\title{
Trends and challenges in open source software (Presentation Video)
}

\section{Stephen Aylward}

Stephen Aylward, "Trends and challenges in open source software (Presentation Video)," Proc. SPIE 8855, Optics and Photonics for Information Processing VII, 885501 (17 October 2013); doi: 10.1117/12.2030336

Event: SPIE Optical Engineering + Applications, 2013, San Diego, California, United States 


\title{
Trends and Challenges in Open Source Software (Presentation Video)
}

\author{
Stephen Aylward, Kitware, Inc. (United States)
}

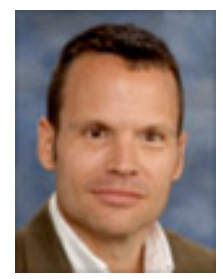

\begin{abstract}
Over the past decade, the field of medical image analysis research has undergone a rapid evolution. It was a collection of disconnected efforts that were burdened by mundane coding and file $\mathrm{I} / \mathrm{O}$ tasks. It is now a collaborative community that has embraced open-source software as a shared foundation, reducing mundane coding and I/O burdens, promoting replicable research, and accelerating the pace of research and product development. This talk will review the history and current state of open-source software in medical image analysis research, will discuss the role of intellectual property in research, and will present emerging trends and technologies relevant to the growing importance of open-source software.
\end{abstract}

View presentation video on SPIE's Digital Library: http://dx.doi.org/10.1117/12.2030336 